\title{
Programmable Transponder, Code and Differentiated Filter Configuration in Elastic Optical Networks
}

\author{
Nicola Sambo, Gianluca Meloni, Francesco Paolucci, Filippo Cugini, \\ Marco Secondini, Francesco Fresi, Luca Potì and Piero Castoldi
}

\begin{abstract}
Next generation optical networks will require high levels of flexibility both at the data and control planes, being able to fit rate, bandwidth, and optical reach requirements of different connections. Optical transmission should be able to support very high rates (e.g., $1 \mathrm{~Tb} / \mathrm{s})$ and to be distance adaptive while optimizing spectral efficiency (i.e., the information rate transmitted over a given bandwidth). Similarly, the control plane should be capable of performing effective routing and spectrum assignment as well as proper selection of the transmission parameters (e.g., modulation format) depending on the required optical reach.
\end{abstract}

In this paper we present and demonstrate a software-defined super-channel transmission based on time frequency packing and on the proposed differentiated filter configuration. Time frequency packing is a technique able to achieve high spectral efficiency even with low-order modulation formats (e.g., quadrature phaseshift keying). It consists in sending pulses that overlap in time or frequency or both to achieve high spectral efficiency. Coding and detection are properly designed to account for the introduced inter-symbol and inter-carrier interference. We present a Software Defined Network (SDN) controller that sets transmission parameters (e.g., code rate) both at the transmitter and the receiver side. In particular, at the transmitter side, a programmable encoder adding redundancy to the data is controlled by SDN. At the receiver side, the digital signal processing is set by SDN based on the selected transmission parameters (e.g., code rate). Thus, extensions to the OpenFlow architectures are presented to control super-channel transmission based on time frequency packing. Then, the SDN-based differentiated filter configuration (DFC) is proposed. According to DFC, the passband of the filters traversed by the same connection can be configured to different values. Experiments including data and control planes are shown to demonstrate the feasibility of optical-reach-adaptive super-channel at $1 \mathrm{~Tb} / \mathrm{s}$ controlled by extended OpenFlow. Then, the effectiveness of the proposed SDN-based DFC is demonstrated in a testbed with both wavelength selective switches and spectrum selective switches, where filters traversed by a connection requires different passband values. Extended OpenFlow messages for time frequency packing and supporting DFC have been captured and shown in the paper.

Index Terms-Software defined network (SDN), OpenFlow, time frequency packing, flexible grid, flexi grid, coding, filter.

Manuscript received April 22, 2014.

N. Sambo (email: nicola.sambo@sssup.it), F. Paolucci, M. Secondini, F. Fresi, and P. Castoldi are with Scuola Superiore Sant'Anna, Via Moruzzi 1, Pisa, Italy. F. Cugini, G. Meloni, and L. Potì are with CNIT, Via Moruzzi 1 , Pisa, Italy.

The authors acknowledge the contribution of G. Colavolpe and T. Foggi who designed the modulation and coding formats based on time frequency packing employed in this paper and also contributed to the implementation of the digital processing employed at the receiver.

This work was partially supported by the FP-7 IDEALIST project under grant agreement number 317999 and the Italian Ministry for Education University and Research (MIUR) under the Firb project COTONE.

\section{INTRODUCTION}

$\mathbf{N}$ EXT generation backbone optical infrastructures will require high level of flexibility (or elasticity) and programmability both at the data- and control-plane levels [1][9]. First, transponders should be able to elastically adapt transmission parameters based on traffic requests. As an example, transmission characteristics should be set based on the path, with the objective of optimizing spectral efficiency while satisfying the required level of quality of transmission (e.g., measured with bit error rate -BER). Currently, several works [1], [10]-[13] have proposed to select the proper modulation format based on the physical characteristics of the path: e.g., using more spectrally efficient modulation formats when supported by the path (e.g., polarization-multiplexing 16-quadrature amplitude modulation - PM-16QAM - along short distances, typically few hundreds of kilometers), while to use more robust formats (e.g., polarization-multiplexing quadrature phase-shift keying -PM-QPSK) for longer distances. Such solutions may require transponders able to support several modulation formats (e.g., PM-16QAM, PMQPSK) [12], so that the control plane can set the transponder according to the chosen format [10], [13].

Alternatively or complementary with the modulation format adaptation, specific data coding can be adopted based on transmission characteristics, as done in the recently introduced time-frequency-packing technique (an evolution of faster-thanNyquist [14]-[17]). In particular, time frequency packing is a transmission technique which consists in sending pulses that strongly overlap in time or frequency or both to maximize spectral efficiency, while introducing inter-symbol and inter-carrier interference ${ }^{1}$. Coding and detection are properly designed to account for the introduced interference. Time frequency packing provides high flexibility because the required all-optical reach can be achieved through the selection of specific code and code rate (e.g., higher redundancy is required for longer paths) even without requiring transponders supporting multiple modulation formats (e.g., supporting PMQPSK).

Another level of flexibility lies in the possibility to program the information rate of the transponders in order to satisfy the dynamics of traffic in terms of amount of transmitted data [1], [12], [18], [19]. Flexibility or elasticity has been also studied in terms of cognitive capabilities, i.e. the capacity of

\footnotetext{
${ }^{1}$ From the information theory point of view adopted here, spectral efficiency is defined as the maximum rate at which information can be reliably transmitted on a given bandwidth, thus accounting also for the introduced interference
} 


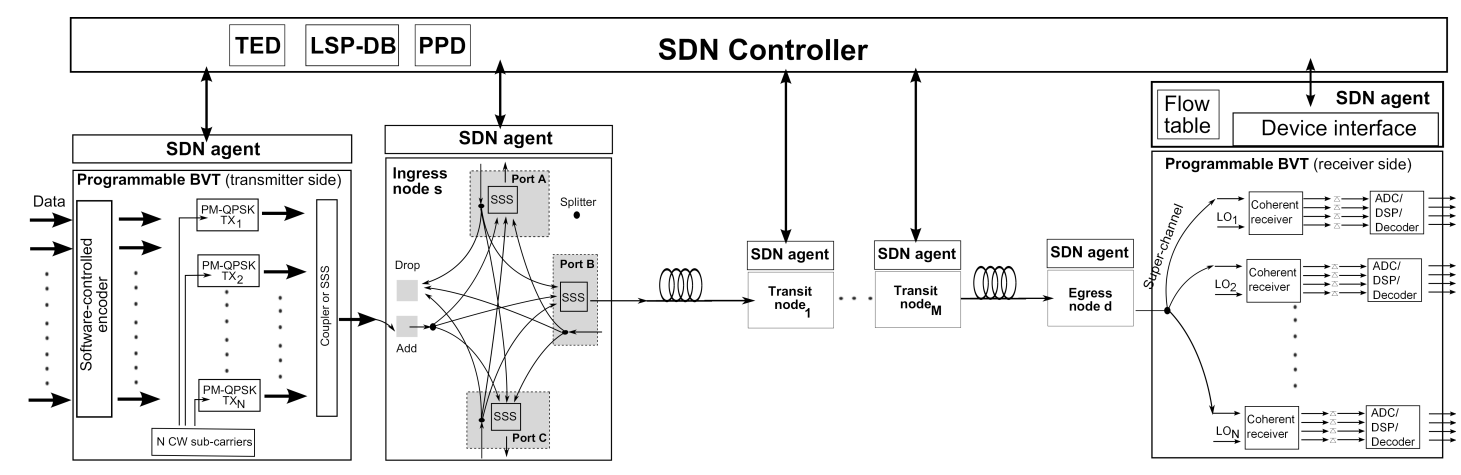

Figure 1. Architecture including BVTs supporting time frequency packing and SDN for transponder and node configuration.

sensing network conditions and reacting according to them with the aim of improving performance (e.g., transmission performance) [20]. Elasticity can be also satisfied at the switching level thanks to the availability of spectrum selective switches (SSSs) [21] and the emerging ITU-T Flex Grid [22]. In particular, the optical filter passband at each node can be configured based on the effective bandwidth required by the traversing optical connections, thus optimizing spectral efficiency [1]. The importance of flexibility is also underlined in [5] where a model to quantitatively evaluate the flexibility provided by optical node components, subsystems, and architectures is proposed. The same work highlights the benefits of node architectures that flexibly adapt their structure based on the current traffic demands.

At the control plane layer, Software Defined Networking (SDN) represents an innovative and attractive paradigm for the control of network infrastructures. The main characteristics of SDN reside in the decoupling of data and control planes with the availability of a centralized controller performing both routing and node configuration. Such centralization enables efficient computations (e.g., avoiding resource contentions [23]) and relaxes the complexity of the network avoiding distributed path computation modules (as in the generalized multi protocol label switching - GMPLS) or the flooding of traffic engineering information within several nodes of the control plane (as in the case of the Open Shortest Path First with Traffic Engineering extensions). Moreover, SDN may decrease lightpath set-up time being node configuration done in parallel instead of sequentially as in the case of GMPLS [24][26]. Currently, the OpenFlow architecture represents the most considered solution for SDN. Although originally designed for Ethernet networks, several studies have recently considered OpenFlow in the context of optical networks [25], [27][31]. In particular, authors in [27] introduced cross-connection tables similar to the flow table in standard OpenFlow, in order to maintain all the cross-connection information within SSSs. In [29], the OpenFlow control of a multi-domain optical network testbed employing packet, fixed and flexible grid technologies is demonstrated. In [28], monitoring functionalities are introduced in the OpenFlow architecture in flex-grid optical networks. In [30], [31], the main principles to extend SDN for optical transport networks are reviewed.

At the state of the art, the control and the programmability of coding has not been addressed in the literature, espe- cially considering the important role of coding in promising transmission techniques such as time frequency packing [15]. Moreover, flexibility in terms of differentiating the configuration of filters traversed by the same connection has not been proposed and investigated.

In this paper we present and demonstrate a softwaredefined super-channel transmission based on time frequency packing and on the proposed differentiated filter configuration. The SDN controller sets transmission parameters both at the transmitter and the receiver side and performs nodes' configuration. At the transmitter side, a programmable transponder generating $N$ PM-QSPK sub-carriers with transmission parameters required by time frequency packing is considered. A programmable encoder controlled by the SDN performs data encoding before sub-carrier modulation. The code rate is controlled via software by SDN. At the receiver side, SDN sets the coherent receiver based on the transmitted signal, thus sets the local oscillators and the receiver parameters, including decoder. Extensions to the OpenFlow architectures are presented to control super-channel transponder based on time frequency packing. Then, SDN also controls the configuration of the nodes, e.g. filter passband. To this purpose, we propose the differentiated filter configuration $(D F C)$ based on SDN. In particular, the passband of the filters traversed by the same connection can be set to different values. This is particularly effective in the presence of detrimental filtering effects or in case of filters based on different technologies (e.g., SSSs or fixed-grid wavelength selective switches -WSSs). Experiments including data and control planes are presented to demonstrate the feasibility of optical-reach-adaptive superchannel at $1 \mathrm{~Tb} / \mathrm{s}$ controlled by extended OpenFlow. Then, an experiment in a testbed including both WSSs and SSSs is carried out to demonstrate the effectiveness of the proposed SDN-based DFC when filters along a connection require different passband values. OpenFlow messages extended for time frequency packing and supporting DFC are captured and shown in the paper.

\section{Architecture based on SDN AND PRogrammable TIME FREQUENCY PACKING TRANSMISSION}

The architecture is summarized in Fig. 1. A centralized SDN controller controls the data plane- i.e. bandwidth variable transponders (BVTs, i.e., transponders requiring different bandwidth values depending on rate, coding, etc.) both at the 
transmitter and receiver side, and node (based on SSSs in the example) configuration. Upon lightpath request, the SDN controller performs path computation and selects the proper transmission parameters (as it will be detailed, together with the SDN architecture, in Sec. V and VI). Then, transmitter, receiver and nodes along the path are configured in parallel in a centralized manner [17], [26]. The transponder at the transmitter side supports time frequency packing and can generate one or multiple carriers (i.e., a super-channel of $N$ sub-carriers). The transponder at the transmitter side consists in a programmable encoder, $N$ lasers, each one generating a continuous wave (CW) sub-carrier ${ }^{2}, N$ PM-QPSK transmitters (one per sub-carrier), and a coupler (or a SSS) to multiplex the sub-carriers. SDN is extended to configure transmission parameters as will be detailed in Secs. III and V. The transponder is connected to the node through the add and drop modules at the transmitter or receiver side, respectively. Regarding the add and drop modules, the architectures presented in [33] can be considered. As a possible node architecture, in this study we consider the one based on broadcast and select [34]. Thus, any signal coming into the node is split and broadcasted to all the ports. Then, the signal is selected by a specific port through the proper configuration of the SSS passband, while it is blocked by the SSSs of the other ports. As an example, considering a super-channel injected in the ingress node, the SSS of Port B selects the super-channel, while the other SSSs block it. In the case the node is based on fixed-grid WSSs, the WSSs would replace the SSSs depicted for the ingress node. Then, the signal traverses several transit nodes (e.g., based on broadcast and select as the ingress node) and reaches the egress node where the super-channel is dropped and received. SDN is designed to configure node ports (i.e., filter passband according to the proposed DFC), as it will be detailed in Secs. IV and V. At the receiver side, the transponder is composed by $N$ coherent receivers, one for each possible sub-carrier. For each coherent receiver, a tunable laser used as local oscillator (LO) must be tuned in order to match the proper sub-carrier. As a result, two optical signals beat into the opto/electronic conversion module providing four analog electrical signals where information is completely mixed in terms of phase, amplitude, and polarization. Analogto-digital (ADC) conversion is performed and digital signal processing (DSP) is used. DSP fully compensates for linear fibre transmission impairments and possibly is designed to implement push-pull defragmentation [35] and digital coherent enhancement [36]. Each receiver includes a decoder for time frequency packing. SDN is extended to configure such transponder as it will be detailed in Secs. III and V.

\section{PROgRAmMABle TRANSMisSiOn BASED ON TIME FREQUENCY PACKING}

A super channel at (gross) line rate $R$ is obtained by transmitting $N$ PM-QPSK optical sub-carriers. The line rate of each sub-carrier is $R_{s}$ and the overall super-channel bandwidth is $B$. Time frequency packing is exploited to reduce

\footnotetext{
${ }^{2}$ Alternatively, the $N$ lasers can be replaced with a multi-wavelength source [32], i.e. a source capable of generating $N \mathrm{CW}$ sub-carriers from a single laser.
}

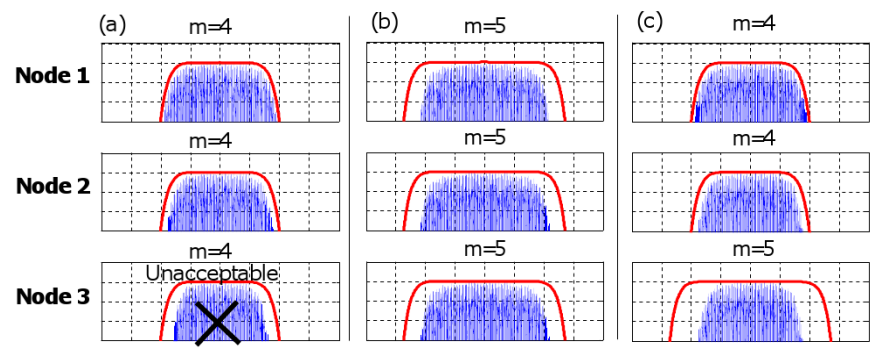

Figure 2. Traditional cascaded filtering with $\mathrm{m}=4(50 \mathrm{GHz}$, unacceptable filtering effects) (a); or $\mathrm{m}=5$ (62.5 GHz, acceptable filtering effects) (b); and Differentiated Filter Configuration (spectrum saving and acceptable filtering effects) (c)

the bandwidth $B_{s}$ of each sub-carrier and their frequency separation $S$ below the Nyquist limit. In particular, $B_{s}$ and $S$ are optimized as explained in [14]. A low-density parity-check (LDPC) code is used to approach the maximum information rate achievable with the given modulation, accounting for the presence of noise, inter-symbol, and inter-carrier interference. Such code is introduced in the transmission thanks to the exploitation of the SDN-controlled encoder in Fig. 1 which enables an adaptive and programmable code rate. Thus the line rate $R$ of the super-channel includes the information rate $R_{I}$ and the code rate $R_{C}$, in particular $R_{I}=R \times R_{C}$. The receiver of each sub-carrier exploits coherent detection with DSP and decoding. In particular, a two dimensional adaptive feed forward equalizer recovers the four signal quadratures, compensating for linear propagation impairments (e.g., groupvelocity dispersion and polarization-mode dispersion) and completing the implementation of the matched filter required for detection. Then, a maximum a posteriori symbol detector, which takes the form of the well-known Bahl-Cocke-JelinekRaviv (BCJR) detector [37], iteratively exchanges information with an LDPC decoder according to the turbo principle [38].

As an example $R=1.12 \mathrm{~Tb} / \mathrm{s}$ can be achieved with $N=7$ PM-QPSK sub-carriers at $R_{s}=160 \mathrm{~Gb} / \mathrm{s}$ (40 Gbaud) and $S=28 \mathrm{GHz}$. Several LDPC code rates can be exploited depending on the physical characteristics of the path (e.g., optical signal to noise ratio -OSNR). In this paper, we assume the set $\{3 / 4,5 / 6,8 / 9,9 / 10\}$ of code rates, thus with corresponding information rates of $\sim\{0.84,0.93,1.0,1.01\}$ $\mathrm{Tb} / \mathrm{s}$. The code rate can be simply set through software in the SDN-controlled encoder. In particular a generic code rate $R_{C}=i / b$ consists in $(b-i)$ redundancy bits added to each $i$ bits of information.

\section{DifFEREnTIATED Filter CONFIGURATION}

The control of ingress, transit and egress nodes requires the configuration of the output ports and the related filters' passband. This is achieved by setting the proper effective frequency slot [22] along the path. The frequency slot is defined as the frequency range allocated for a connection, within the flexible grid and unavailable to other slots [22], [39]. A frequency slot is identified by its nominal central frequency and its slot width. The nominal central frequency $f$ of a frequency slot is identified by the parameter $n$, such that $\mathrm{f}=193.1 \mathrm{THz}+\mathrm{n} \times 0.00625 \mathrm{THz}$, where $n$ is a positive or negative integer including 0 . The slot width (equal or higher 
than the required bandwidth) indicates the amount of reserved optical spectrum and it is defined to be $\mathrm{m} \times 12.5 \mathrm{GHz}$, where $m$ is an integer greater than or equal to 1 . The frequency slot of a lightpath has to be computed according to the aforementioned transmission parameters (e.g., number of sub-carriers in Sec. III) and the expected quality of transmission (QoT), which should also account for filtering effects in the nodes [40] (e.g., through an OSNR penalty [41]). Indeed, filters present non-ideal rectangular behaviour, with non-negligible transition bands that may introduce distortions on the transmitted signal. So far, the frequency slot is configured by assigning a unique couple $(n, m)$ along the entire connection, i.e. the same bandwidth is configured in all the traversed filters. Fig. 2 illustrates an example of connection transmission through a cascade of three nodes. First, it is assumed that $m=4$ is assigned to the connection (Fig. 2a). It is also assumed that after the first and second node, acceptable QoT is experienced. However, after the third node, excessive detrimental filtering effects are experienced, preventing the actual setup along the three nodes with $m=4$. Thus, larger bandwidth should be computed and configured in order to operate filtering in a more flat region (i.e., avoiding the filter transition bands), thus limiting filtering effects. Fig. $2 b$ shows the frequency slot configured, in all the three nodes, with $\mathrm{m}=5$. In this case, adequate QoT is achieved, at the expenses of more reserved spectrum in all traversed nodes.

Fig. 2c shows the proposed Differentiated Filter Configuration (DFC) solution. In DFC, $m$ can be configured with different values along the path, i.e. different effective bandwidth is configured in the filters traversed by the same connection. In particular, the first and second node are configured with $m=4$, while $m=5$ is applied to last node. This way, no additional detrimental filtering effects are introduced by the third node, and in particular by its filter, which is traversed by the lightpath in its flat region. As a result, spectrum reservation can be minimized on a per node basis according to the expected QoT, thus improving the overall spectrum utilization. Such a technique can be applied both to single carrier and super-channel. Filtering effects can be considered through analytical models (e.g., [41]) or measurements (as we did in Sec. VI).

In a GMPLS-controlled optical network, the $(n, m)$ couple is included within the RSVP-TE protocol and it is unique for all traversed nodes (i.e., as in the traditional case of Fig. 2a, b). Thus, RSVP-TE protocol sequentially configures the passband of filters along the path using the same $(n, m)$. In particular, the requested effective frequency slot is included within the RSVP-TE TSpec and the reserved effective frequency slot is mapped into the RSVP-TE FlowSpec [39]. The implementation of the proposed DFC in the context of GMPLS, although feasible, would then require significant changes in the protocol implementation. For example, this could be achieved by removing these TSpec and FlowSpec extensions and by introducing new $(n, m)$-related extensions within the Explicit Route Object (ERO).

On the other hand, as shown in Sec. VI, the implementation of the proposed DFC is extremely suitable for the SDN architecture, given the possibility to directly and independently control, besides the ingress and egress nodes, also any intermediate node through the specific effective frequency slot and filtering parameters.

\section{SDN ARCHITECTURE}

The proposed SDN control plane is based on extended OpenFlow architecture. A request for a super-channel at information rate $R_{I}$ from ingress $s$ to egress $d$ is considered. The SDN agent at $s$ (see Fig. 1) sends a OFPT_LIGHTPATH_IN message (an extended version of OFPT_PACKET_IN for flex-grid [27], [28]) to the OpenFlow (OF) controller (e.g., Nox) to request both computation and connection set up. In particular, the OFPT_LIGHTPATH_IN message encloses all the lightpath request parameters, such as the requested bit rate and the end-points. Upon message reception, the OF-controller computes the path $p_{s, d}$ connecting $s$ and $d$. OF-controller exploits Traffic Engineering Database (TED) and Label Switch Path Database (LSP-DB) for path computation [42] (see Fig. 1). Then, the OF-controller also exploits a physical path database (PPD) to have knowledge of the quality of transmission (QoT) - e.g., BER - experienced by the superchannel along $p_{s, d}$ when specific transmission parameters are adopted. PPD includes physical network information (e.g., fiber type), it can be filled during network installation and can be updated based on monitored parameter information. In particular, the OF-controller is here enhanced to select, depending on the path (e.g., optical reach), a specific code rate $R_{C}$ which guarantees the required QoT (e.g., error free). In this study, the relation between the computed path $p_{s, d}$ and the adopted code rate, as detailed in the next section, is based on performed measurements. Alternatively, proper models could be adopted to determine the achievable information rate on a given path [43] and to select the code accordingly. Upon code rate selection, the OF-controller computes $R$. Then, based on the electronics at the transmitter and at the receiver, which drives the maximum rate $R_{s}$ of each sub-carrier, the OFcontroller also computes $N$ to achieve $R$. As an example, assuming that the electronics is designed to operate with 40 Gbaud, $R_{s}=160 \mathrm{~Gb} / \mathrm{s}, N=7$ would serve $R=1.12 \mathrm{~Tb} / \mathrm{s}$. Finally, the OF-controller computes $B_{s}, S$ and $B$. Then, the OF-controller acts to configure transmission parameters at the transmitter and receiver and to configure the nodes along $p_{s, d}$, according to DFC. In particular, the OF-Controller sends an extended OFPT_FLOW_MOD to all the SDN agents along $p_{s, d}$, including the ones related to the transponders at the transmitter and receiver sides. SDN agents update the flow table (e.g., cross connections in the node) and configure the corresponding device in the data plane by relying on proper dataplane interfaces (as summarized in Fig. 1).

The OFPT_FLOW_MOD messages directed to the intermediate nodes are extended for flex-grid networks. Such extensions aims at controlling the SSSs, allocating or releasing cross-connections defined with parameters relevant in flex-grid contexts, e.g. central frequency, slot width, modulation format. According to DFC, for the same connection, OFPT_FLOW_MOD messages, directed to the different nodes along the path, can carry different slot width values. Moreover, a new OpenFlow struct is proposed to be intro- 


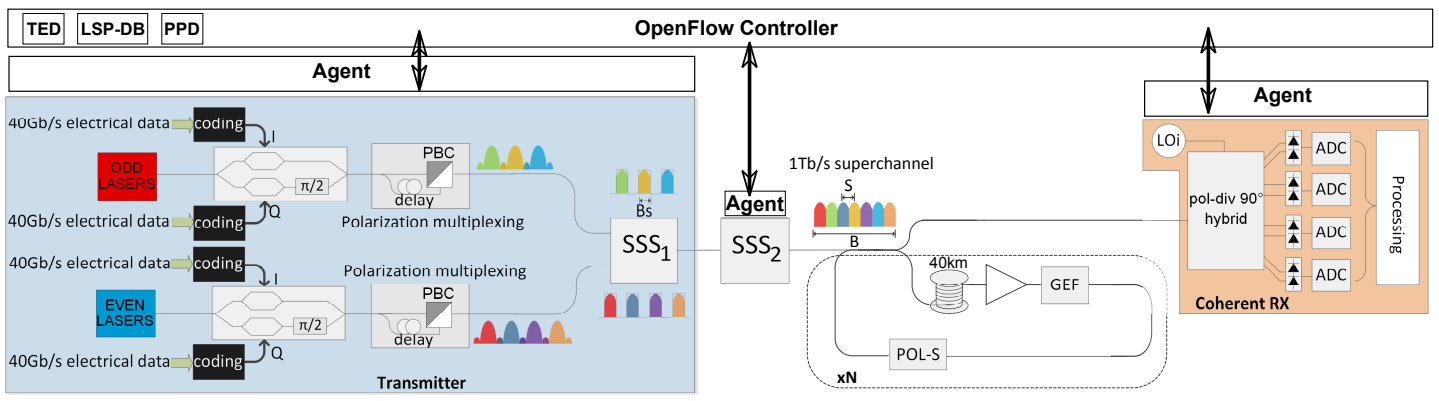

Figure 3. Testbed.

duced in the OFPT_FLOW_MOD messages directed to $s$ and $d$. The new structure, called Optical Channel Specification (OFPT_OCH_SPEC), advertises that time frequency packing technique has to be exploited. The OFPT_OCH_SPEC includes:

1) type of channel (e.g., single-carrier, super-channel);

2) number of sub carriers $N$;

3) subcarrier bit rate $R_{s}$;

4) sub-carrier modulation format;

5) sub-carrier central frequency;

6) sub-carrier bandwidth $B_{s}$;

7) Nyquist flag (i.e, Nyquist, or time frequency packing) sub carrier;

8) the type of adopted code (e.g., in case of time frequency packing);

9) the code rate.

This way, the transmitter at $s$ is configured with the required transmission parameters. Besides the aforementioned fields, the OFPT_OCH_SPEC directed to the egress node will also include a field to describe the type of detection, i.e. symbolby-symbol, or BCJR for time frequency packing. Thus, the coherent receiver at $d$ can be adequately configured with proper detection and decoding parameters (e.g., code rate). Finally, $s, d$, and intermediate nodes send to the OF-controller a OFPT_ACK to notify that a correct configuration of the transmitter, receiver, and filters has been performed.

\section{EXPERIMENTAL DEMONSTRATION}

Several experiments have been carried out to demonstrate the SDN-controlled transmission based on time frequency packing and DFC. OpenFlow protocol has been extended starting from standard version 1.3. The OF-controller is interfaced to the Agents by means of Gigabit Ethernet interfaces. The agents are interfaced to the SSSs (or WSSs) by means of USB 2.0 interface, and to the transmitter and the receiver hardware modules by means of serial/GPIB interfaces. In this study, the implementation of PPD and of the enhanced OF-controller performing path and transmission parameter computation is based on measurements. In particular, we correlated the physical characteristics of the path (e.g., length) with a code rate guaranteeing error free transmission.

To assess the proposed programmable code-rate transmission and DFC, three experiments are performed. The first two experiments are carried out in a flex-grid scenario, while the third experiment in a mixed scenario including both fixedgrid WSSs and flex-grid SSSs. The first experiment aims at analyzing a transmission where the overall super-channel line rate $R$ is fixed and the code rate could change depending on the optical path, thus implying a change in the information rate. Conversely, the second experiment aims at evaluating the transmission when the information rate $R_{I}$ is preserved under different optical path conditions (thus changing $R$ based on the code rate). The two experiments show the experienced relation between $R, R_{I}$, the path, the code-rate and $B$. The third experiment aims at demonstrating the ability of the proposed SDN-based DFC in differentiating filter configuration, considering WSS and SSS.

In the first experiment, a super-channel with $R=1.12 \mathrm{~Tb} / \mathrm{s}$, $N=7, S=28 \mathrm{GHz}$, and $B=200 \mathrm{GHz}$ has been generated. The measurement aims at investigating which is the most effective code rate that achieves error free transmission, for a given all-optical reach. The testbed at the data plane is reported in Fig. 3. A recirculating loop is exploited to vary the all-optical reach. $N=7$ optical carriers are generated by means of $100 \mathrm{kHz}$ linewidth tunable laser sources (TLSs). In particular, the odd an even channels are modulated separately by means of two integrated double nested Mach Zehnder modulators (IQ-MZM). $40 \mathrm{~Gb} / \mathrm{s} \mathrm{LDPC-coded} \mathrm{electrical} \mathrm{signals}$ are applied to the in-phase (I) and the quadrature (Q) port of the modulators. This way, $80 \mathrm{~Gb} / \mathrm{s}$ QPSK channels are obtained. The bit rate is then further doubled up to $160 \mathrm{~Gb} / \mathrm{s}$ per channel, by emulating polarization multiplexing through a 50/50 beam splitter, an optical delay, and a polarization beam combiner (PBC). A first programmable SSS ( $\left.\mathrm{SSS}_{1}\right)$ is used to separately filter and multiplex the odd and the even channels. The optical spectra of the generated super-channel is reported in Fig. 4. The generated signal enters the ingress node which is emulated by the second SSS $\left(\mathrm{SSS}_{2}\right)$, then it is transmitted through a recirculating fiber loop (used to emulate the signal transmission over a real link) by means of a 50/50 optical beam splitter. The recirculating loop is composed by a $40 \mathrm{~km}$-long standard single mode fiber spool followed by an EDFA and a gain equalized filter (GEF). A polarization scrambler (POL-S) in the loop emulates random signal polarization variation.

Given the path length, the highest code rate (i.e., lowest redundancy) guaranteeing error-free transmission after forward error correction is selected. Based on the code rate and the fixed $R$, the information rate $R_{I}$ is derived. Fig. 5 shows the information rate versus the path length. Thus, each path length in the plot is associated with the suitable code rate. Then, given the selected code rate, the information rate is shown. As an example, for a path length of $3000 \mathrm{~km}$, a code rate 


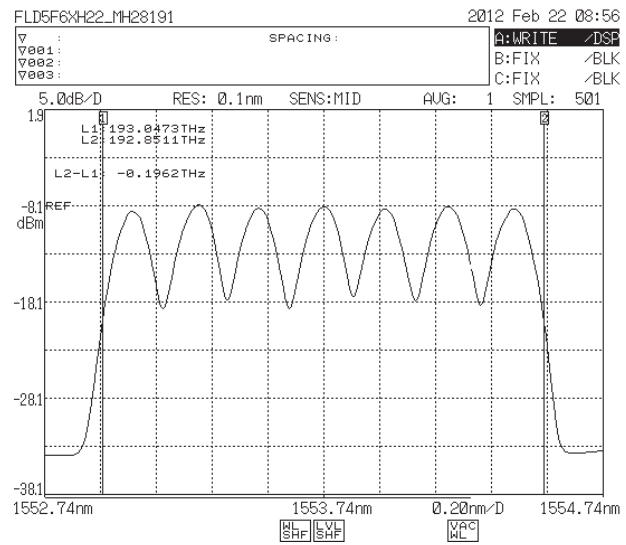

Figure 4. Optical spectrum of the super-channel.

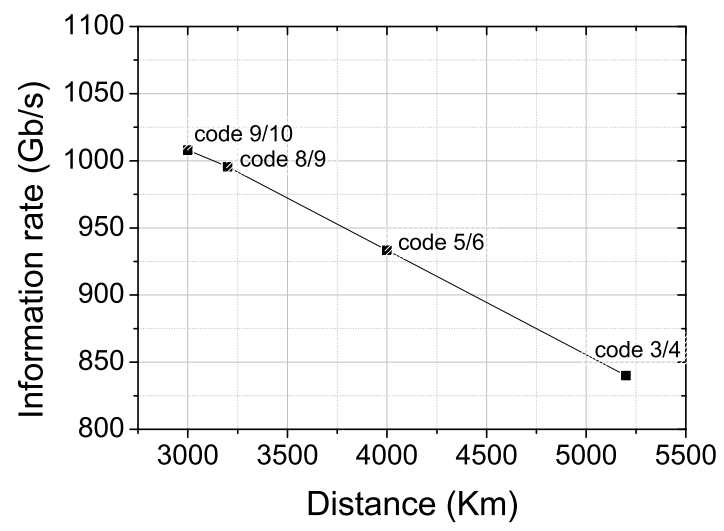

Figure 5. $R_{I}$ vs. optical reach when $\mathrm{R}=1.12 \mathrm{~Tb} / \mathrm{s}, \mathrm{N}=7$, and $\mathrm{B}=200 \mathrm{GHz}$.

of $9 / 10$ assures error-free transmission. With $9 / 10$ code rate, $\sim 1 \mathrm{~Tb} / \mathrm{s}$ of information rate is achieved. Fig. 5 shows that the redundancy of code increases with the path length because more robustness to the impairments is required. Consequently, information rate decreases with the path length if $R$ is fixed. For example, an information rate of $1 \mathrm{~Tb} / \mathrm{s}$ can be assured for a path length of $3000 \mathrm{~km}$, while for a length of $5250 \mathrm{~km}$ information rate is reduced by $\sim 17 \%$ with respect to $1 \mathrm{~Tb} / \mathrm{s}$.

In the second experiment, $R_{I}$ is fixed to $1 \mathrm{~Tb} / \mathrm{s}$. Fig. 6 shows the number of 40 Gbaud PM-QPSK sub-carriers required to fulfill the required $R_{I}$, versus the all-optical reach. Since the required redundancy increases with the all-optical reach and $\mathrm{R}_{\mathrm{I}}$ is fixed, $R$ increases with the all-optical reach. Consequently, more carriers are needed to fulfill the line rate increase, being the maximum sub-carrier baud rate fixed by the electronics. Thus, $N$ increases with the all-optical reach (as shown in Fig. 6). As an example, $N=8$ is required to support $R_{I}=1 \mathrm{~Tb} / \mathrm{s}$ and the code rate of $5 / 6$, that guarantees error free within 3000 and $4000 \mathrm{~km}$ of all-optical reach. Fig. 6 also shows the related bandwidth requirements with a $S=28 \mathrm{GHz}$ for the code rate guaranteeing error free transmission. $B$ increases with the all-optical reach since $R$ increases. Note that for a distance shorter than $3000 \mathrm{~km}$ a spectral efficiency of $5.14 \mathrm{~b} / \mathrm{s} / \mathrm{Hz}$ is obtained, thus a value larger than the maximum one theoretically achievable by a PM-QPSK Nyquist transmission (4 bit/s/Hz).

The testbed used for the third experiment is derived from

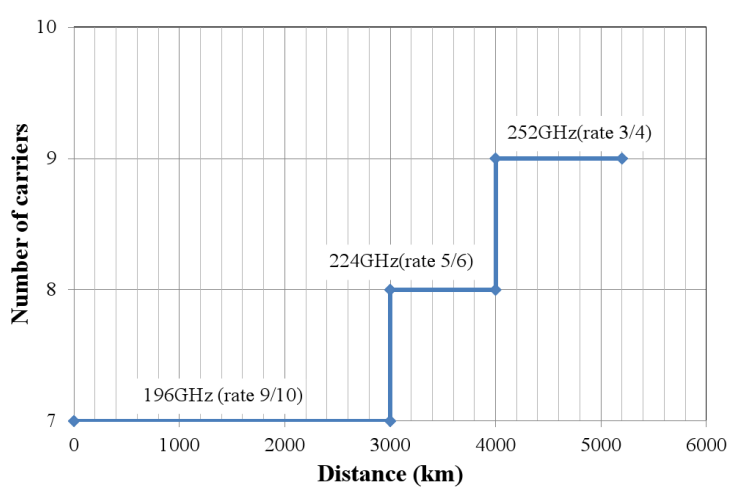

Figure 6. $\mathrm{N}$ and $\mathrm{B}$ vs. all-optical reach with $\mathrm{R}_{\mathrm{I}}=1 \mathrm{~Tb} / \mathrm{s}$ and $\mathrm{S}=28 \mathrm{GHz}$.

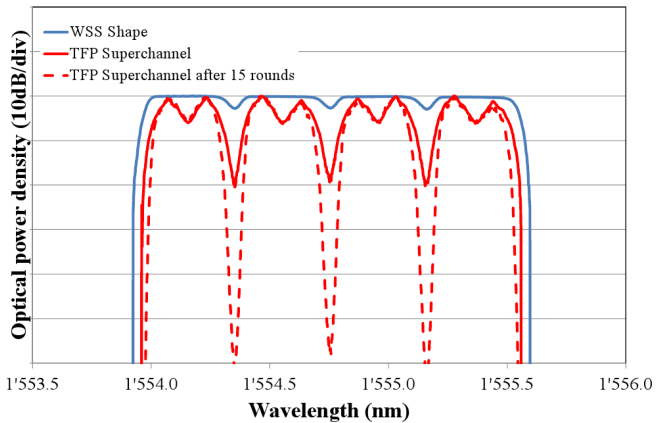

Figure 7. Spectrum of the $1 \mathrm{~Tb} / \mathrm{s}$ in the presence of WSSs.

the one in Fig. 3. In particular, a $50 \mathrm{GHz}$-fixed grid WSS is inserted between the EDFA and the GEF of the recirculating loop. To fit the fixed grid, a $1 \mathrm{~Tb} / \mathrm{s}$ super-channel has been designed as in Fig. 7, that shows the WSS filter shape, the spectrum of the generated super-channel, and its spectrum after 15 loops. In this case, the super-channel is achieved with $N=8$. Sub-carrier spacing has been designed such that two sub-carriers can be accommodated within the 50$\mathrm{GHz}$ fixed grid. Consequently, the super-channel can occupy $4 \times 50 \mathrm{GHz}=200 \mathrm{GHz}$. The baud rate of each sub-carrier has been set to 35 or 40 Gbaud. Fig. 8 shows the information rate vs. the number of crossed WSSs for different values of carrier baud rate. During the experiment, the passband of $\mathrm{SSS}_{2}$ at the ingress node was set to $225 \mathrm{GHz}$ (i.e., larger than $200 \mathrm{GHz}$ ) thus operating filtering in the flat region and resulting in negligible SSS filtering effects. The maximum number of WSSs experimentally crossed is 15 at $4.2 \mathrm{~b} / \mathrm{s} / \mathrm{Hz}$ and 35 Gbaud $\left(R_{C}=3 / 4, R_{I}=0.84 \mathrm{~Tb} / \mathrm{s}\right)$. On the other side, at a spectral efficiency of $5.69 \mathrm{~b} / \mathrm{s} / \mathrm{Hz}$ and 40 Gbaud $\left(R_{C}=8 / 9, R_{I}=1.138 \mathrm{~Tb} / \mathrm{s}\right)$ up to $5 \mathrm{WSSs}$ could be crossed.

Transmission parameter information derived from such experiments have been uploaded in the PPD exploited by the implemented OF-controller in order to select the proper transmission parameters upon connection request. The OFcontroller performs path computation, then, by exploiting PPD, selects proper transmission parameters (e.g., code rate) to guarantee error free transmission along the computed path. First, a request of $R_{I}=1 \mathrm{~Tb} / \mathrm{s}$ is considered in a flex-grid scenario. OpenFlow messages are exchanged in the control plane. The OF-controller processes the request computing a path of $3250 \mathrm{~km}$. Based on measurements in Fig. 6, the OF- 
Controller computes the proper transmission parameters (e.g., $R_{C}=5 / 6, N=8$ ). Fig. 9 shows the OpenFlow messages exchanged within the control plane between the OpenFlow Controller (IP 10.0.0.49) and the OpenFlow switch agents (IP 10.0.0.x, $x \in[1,4]$ ) that are co-located within the four testbed nodes and are responsible of configuring the transmitter/receiver physical parameters and the SSS filter shapes. In particular, Fig. 9 shows a capture of the OFPT_FLOW_MOD message sent to the ingress node. The OFPT_FLOW_MOD message includes the proposed OFPT_OCH_SPEC OpenFlow struct for time frequency packing. As shown in the figure the struct includes: 1) type of channel: multi-carrier; 2) $N=8$; 3) $R_{s}=160 \mathrm{~Gb} / \mathrm{s}$; 4) sub-carrier modulation format: PM-QPSK each; 5) sub-carrier central frequency (e.g., $192.894 \mathrm{GHz}$ ); 6) $B_{s}=28 \mathrm{GHz}$; 7) Nyquist flag: time frequency packing; 8) the type of adopted code: LDPC; 9) $R_{C}=5 / 6$ for a path length of $3250 \mathrm{~km}$. Moreover, the $\mathrm{SSS}_{2}$ is set to $n=-22$ and $m=18$ for the super-channel pass-through.

Then, control plane message capture is also performed in the mixed fixed/flex-grid scenario to demonstrate the effectiveness of SDN-based DFC. A request of $R_{I}=1 \mathrm{~Tb} / \mathrm{s}$ is assumed. The OF-controller processes the request computing a path of thirteen nodes, thus including $\mathrm{SSS}_{2}$ at the ingress and twelve WSSs in the other nodes (each one emulated in the recirculating loop). Transmission parameters are selected (e.g., 35 Gbaud corresponding to $R_{s}=140 \mathrm{~Gb} / \mathrm{s}$ and to $R_{C}=8 / 9$ ) based on measurements in Fig. 8, obtained with a $\mathrm{SSS}_{2}$ passband of $225 \mathrm{GHz}$ and WSS passbands of $4 \times 50 \mathrm{GHz}$ (as in Fig. 7). Fig. 10 shows two OFPT_FLOW_MOD messages sent to the ingress node equipped with a SSS (packet 99) and to an intermediate node equipped with $50 \mathrm{GHz}$-fixed grid WSS (packet 100), respectively. The recirculating loop is emulated within the control plane closed around node 2 (IP 10.0.0.2). The former message shows the spectrum label sent to $\mathrm{SSS}_{2}$, employing flexible grid with $m=18$ resulting in $B=225 \mathrm{GHz}$. The latter message shows the extended DWDM label simultaneously activating at the WSS four adjacent $50 \mathrm{GHz}$ standard channels in order to shape the super-channel properly (as in Fig. 7). The extended label version specifies the edges of the super-channel (expressed as $n$ values with $50 \mathrm{GHz}$ channel spacing, in this case from $n=-7$ to $n=-4$ ) to be activated. The experiment demonstrated that SDN can easily handle DFC, thus differentiating the configuration of filters along the path (SSS to $225 \mathrm{GHz}$ and WSSs to $50 \mathrm{GHz}$ ).

\section{CONCLUSIONS}

We presented and demonstrated a software-defined superchannel transmission based on time frequency packing and on the proposed differentiated filter configuration. First, SDN is extended to support the configuration of parameters (e.g., code rate, number of sub-carriers) both at the transmitter and the receiver side (e.g., decoding at the DSP). At the transmitter side, a programmable software is used to encode data based on time frequency packing and on the selected code rate. At the receiver side, a programmable DSP is capable of elaborating and decoding received data. Differentiated filter configuration (DFC) is also proposed for node configuration. According to DFC, the passband of the filters traversed by the same

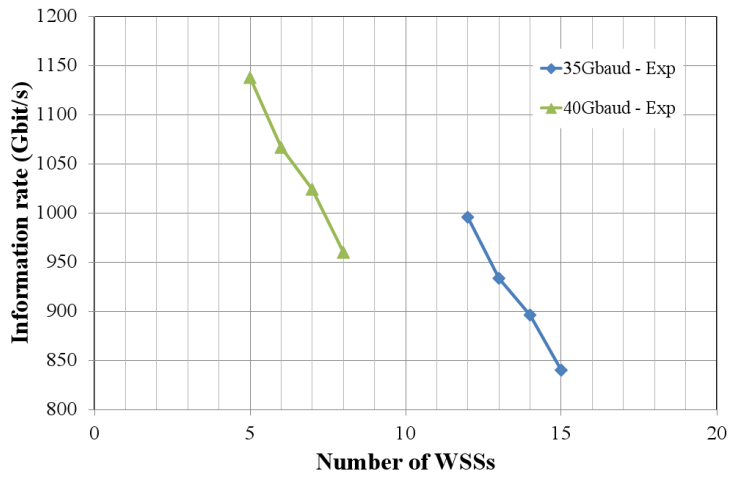

Figure 8. $R_{I}$ vs. number of crossed WSSs with $\mathrm{N}=8$, in a mixed fixed/flexgrid scenario with $\mathrm{SSS}_{2}$ passband set to $225 \mathrm{GHz}$.

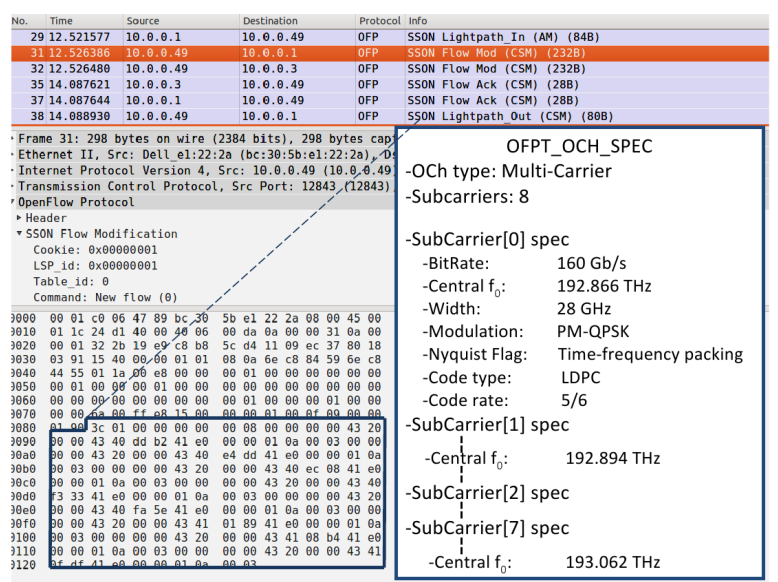

Figure 9. Capture of OpenFlow messages in the flex-grid scenario.

connection can be configured to different values. This can be easily done thanks to the exploitation of SDN which, differently from RSVP-TE, does not require that the passband value is unique for the whole connection. DFC can be particularly effective in case of a network equipped with filters based on different technologies (e.g., WSSs and SSSs) or in the presence of detrimental filtering effects. Experiments including data and control planes have been performed to demonstrate the feasibility of DFC and of optical-reach-adaptive super-channel at $1 \mathrm{~Tb} / \mathrm{s}$ controlled by extended OpenFlow. Distance-adaptive$\mathrm{Tb} / \mathrm{s}$ transmission has been demonstrated both by fixing the line rate (by varying information rate) and by fixing the information rate (by varying the bandwidth). An experiment also demonstrated the effectiveness of SDN-based DFC in differentiating the configuration of filters along the path, also considering different technologies (SSS and WSS).

\section{REFERENCES}

[1] M. Jinno, B. Kozicki, H. Takara, A. Watanabe, Y. Sone, T. Tanaka, and A. Hirano, "Distance-adaptive spectrum resource allocation in spectrumsliced elastic optical path network," Communications Magazine, IEEE, vol. 48, no. 8, pp. $138-145$, august 2010.

[2] O. Gerstel, M. Jinno, A. Lord, and S. Yoo, "Elastic optical networking: a new dawn for the optical layer?" Communications Magazine, IEEE, vol. 50, no. 2, pp. s12-s20, february 2012.

[3] N. Sambo, P. Castoldi, F. Cugini, G. Bottari, and P. Iovanna, "Toward high-rate and flexible optical networks," Communications Magazine, IEEE, vol. 50, no. 5, pp. $66-72$, may 2012.

[4] M. Jinno, H. Takara, Y. Sone, K. Yonenaga, and A. Hirano, "Multiflow optical transponder for efficient multilayer optical networking," Communications Magazine, IEEE, vol. 50, no. 5, pp. 56-65, 2012. 


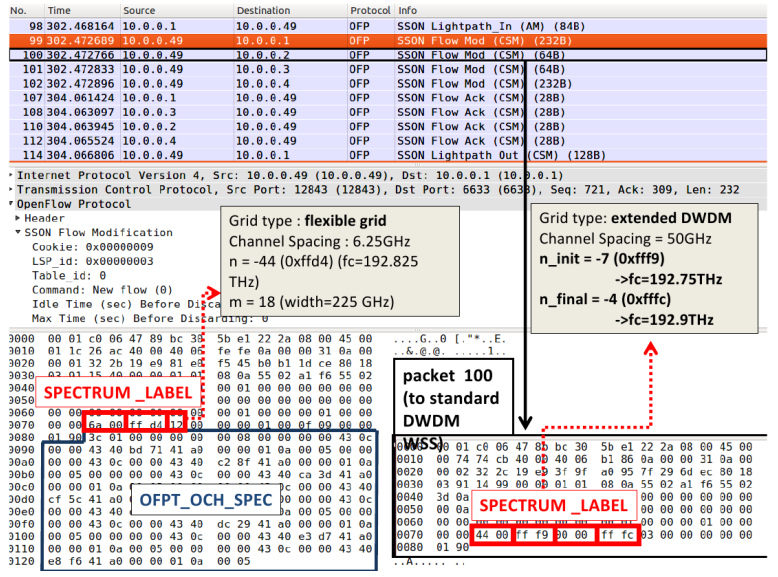

Figure 10. Capture of OpenFlow messages in the fixed/flex-grid scenario.

[5] N. Amaya, G. Zervas, and D. Simeonidou, "Introducing node architecture flexibility for elastic optical networks," Optical Communications and Networking, IEEE/OSA Journal of, vol. 5, no. 6, pp. 593-608, 2013.

[6] M. Klinkowski, M. Ruiz, L. Velasco, D. Careglio, V. Lopez, and J. Comellas, "Elastic spectrum allocation for time-varying traffic in flexgrid optical networks," Selected Areas in Communications, IEEE Journal on, vol. 31, no. 1, pp. 26-38, 2013.

[7] M. Liu, M. Tornatore, and B. Mukherjee, "Survivable traffic grooming in elastic optical networks -shared protection," Lightwave Technology, Journal of, vol. 31, no. 6, pp. 903-909, 2013.

[8] Y. Zhao, X. Yu, Y. Yu, J. Zhang, and L. Wang, "Novel control plane framework and protocol extensions for spectrum-efficient optical transport networks," OSN, vol. 10, no. 3, pp. 211-222, 2013.

[9] B. Rofoee, G. Zervas, Y. Yan, N. Amaya, and D. Simeonidou, "All programmable and synthetic optical network: Architecture and implementation," Optical Communications and Networking, IEEE/OSA Journal of, vol. 5, no. 9, pp. 1096-1110, 2013.

[10] F. Cugini, G. Meloni, F. Paolucci, N. Sambo, M. Secondini, L. Gerardi, L. Poti, and P. Castoldi, "Demonstration of flexible optical network based on path computation element," Lightwave Technology, Journal of, vol. 30, no. 5, pp. $727-733$, march1, 2012.

[11] X. Cai, K. Wen, R. Proietti, Y. Yin, R. Scott, C. Qin, and S. J. B. Yoo, "Experimental demonstration of adaptive combinational QoT failure restoration in flexible bandwidth networks," in Proc. of OFC/NFOEC 2012, 2012.

[12] F. Cugini, F. Paolucci, N. Sambo, L. Potì, A. D’Errico, and G. Bottari, "Reliable flexible-ROADM architecture enabling modulation format adaptation," in Proc. of ECOC 2012, 2012.

[13] L. Liu, H. Y. Choi, R. Casellas, T. Tsuritani, I. Morita, R. Martinez, and R. Munoz, "Demonstration of a dynamic transparent optical network employing flexible transmitters/receivers controlled by an OpenFlowstateless PCE integrated control plane [invited]," Optical Communications and Networking, IEEE/OSA Journal of, vol. 5, no. 10, 2013.

[14] A. Barbieri, D. Fertonani, and G. Colavolpe, "Time-frequency packing for linear modulations: spectral efficiency and practical detection schemes," Communications, IEEE Transactions on, vol. 57, no. 10, 2009.

[15] L. Potì, G. Meloni, G. Berrettini, F. Fresi, M. Secondini, T. Foggi, G. Colavolpe, E. Forestieri, A. D'Errico, F. Cavaliere, R. Sabella, and G. Prati, "Casting $1 \mathrm{~Tb} / \mathrm{s}$ DP-QPSK communication into $200 \mathrm{GHz}$ bandwidth," in Proc. of ECOC 2012, Sep. 2012.

[16] G. Colavolpe and T. Foggi, "High spectral efficiency for long-haul optical links: Time-frequency packing vs high-order constellations," in Proc. of ECOC 2013, 2013.

[17] N. Sambo, F. Paolucci, F. Cugini, M. Secondini, L. Potì, G. Berrettini, G. Meloni, F. Fresi, G. Bottari, and P. Castoldi, "Software defined coderate-adaptive Terabit/s based on time-frequency packing," in Proc. of OFC/NFOEC 2013, March 2013.

[18] G. Zervas, M. De Leenheer, L. Sadeghioon, D. Klonidis, Y. Qin, R. Nejabati, D. Simeonidou, C. Develder, B. Dhoedt, and P. Demeester, "Multigranular optical cross-connect: Design, analysis, and demonstration," Optical Communications and Networking, IEEE/OSA Journal of, vol. 1, no. 1, pp. 69-84, 2009.

[19] H. Takara, T. Goh, K. Shibahara, K. Yonenaga, S. Kawai, and M. Jinno, "Experimental demonstration of $400 \mathrm{~Gb} / \mathrm{s}$ multi-flow, multi-rate, multi- reach optical transmitter for efficient elastic spectral routing," in Proc. of ECOC 2011, 2011.

[20] I. de Miguel et al., "Cognitive dynamic optical networks [invited]," Optical Communications and Networking, IEEE/OSA Journal of, vol. 5, no. 10,2013

[21] in www.finisar.com/products/wss-roadms.

[22] "Draft revised G.694.1 version 1.3," Unpublished ITU-T Study Group 15, Question 6.

[23] A. Giorgetti, F. Cugini, N. Sambo, F. Paolucci, N. Andriolli, and P. Castoldi, "Path state-based update of PCE traffic engineering database in wavelength switched optical networks," Communications Letters, IEEE, vol. 14 , no. 6 , pp. 575-577, 2010

[24] A. Giorgetti, F. Cugini, F. Paolucci, and P. Castoldi, "Openflow and PCE architectures in wavelength switched optical networks," in Proc. of ONDM 2012, 2012.

[25] D. Simeonidou, R. Nejabati, and M. Channegowda, "Software defined optical networks technology and infrastructure: Enabling softwaredefined optical network operations," in Proc. of OFC/NFOEC, 2013.

[26] R. Casellas, R. Martinez, R. Munoz, R. Vilalta, L. Liu, T. Tsuritani, and I. Morita, "Control and management of flexi-grid optical networks with an integrated stateful path computation element and openflow controller [invited]," Optical Communications and Networking, IEEE/OSA Journal of, vol. 5, no. 10, pp. A57-A65, Oct 2013.

[27] L. Liu, R. Muñoz, R. Casellas, T. Tsuritani, R. Martínez, and I. Morita, "Openslice: an openflow-based control plane for spectrum sliced elastic optical path networks," in Proc. of ECOC 2012, 2012.

[28] F. Paolucci, F. Cugini, N. Hussain, F. Fresi, and L. Potì, "Openflow-based flexible optical networks with enhanced monitoring functionalities," in Proc. of ECOC 2012, 2012.

[29] M. Channegowda et al., "First demonstration of an OpenFlow based software-defined optical network employing packet, fixed and flexible DWDM grid technologies on an international multi-domain testbed," in Proc. of ECOC 2012, sept. 2012.

[30] L. Liu, T. Tsuritani, I. Morita, and S. Yoo, "Optical network control and management technology using OpenFlow," in OptoElectronics and Communications Conference held jointly with 2013 International Conference on Photonics in Switching (OECC/PS), 2013 18th, 2013.

[31] S. Gringeri, N. Bitar, and T. Xia, "Extending software defined network principles to include optical transport," Communications Magazine, IEEE, vol. 51, no. 3, pp. 32-40, 2013.

[32] P. Anandarajah, R. Zhou, R. Maher, M. Gutierrez Pascual, F. Smyth, V. Vujicic, and L. Barry, "Flexible optical comb source for super channel systems," in Proc. of OFC/NFOEC 2013, 2013.

[33] P. Roorda and B. Collings, "Evolution to colorless and directionless ROADM architectures," in Proc. of OFC/NFOEC 2008, 2008.

[34] N. Sambo, G. Meloni, G. Berrettini, F. Paolucci, A. Malacarne, A. Bogoni, F. Cugini, L. Poti, and P. Castoldi, "Demonstration of data and control plane for optical multicast at 100 and $200 \mathrm{~Gb} / \mathrm{s}$ with and without frequency conversion," Optical Communications and Networking, IEEE/OSA Journal of, vol. 5, no. 7, pp. 667-676, 2013.

[35] F. Cugini, F. Paolucci, G. Meloni, G. Berrettini, M. Secondini, F. Fresi, N. Sambo, L. Poti, and P. Castoldi, "Push-pull defragmentation without traffic disruption in flexible grid optical networks," Lightwave Technology, Journal of, vol. 31, no. 1, pp. 125-133, 2013.

[36] G. Colavolpe, T. Foggi, E. Forestieri, and M. Secondini, "Impact of Phase Noise and Compensation Techniques in Coherent Optical Systems," J. Lightwave Technol., vol. 29, no. 18.

[37] L. Bahl, J. Cocke, F. Jelinek, and J. Raviv, "Optimal decoding of linear codes for minimizing symbol error rate," Information Theory, IEEE Transactions on, 1974.

[38] J. Hagenauer, "The turbo principle: Tutorial introduction and state of the art," in Proc. International Symposium on Turbo Codes and Related Topics, 1997.

[39] D. King, A. Farrel, Y. Li, F. Zhang, and R. Casellas, IETF draftfarrkingel-ccamp-flexigrid-lambda-label-04, Oct. 2012.

[40] S. Poole, S. Frisken, M. Roelens, and C. Cameron, "Bandwidth-flexible ROADMs as network elements," in Proc. of OFC/NFOEC 2011, 2011.

[41] B. Kozicki, H. Takara, Y. Tsukishima, T. Yoshimatsu, K. Yonenaga, and M. Jinno, "Experimental demonstration of spectrum-sliced elastic optical path network (SLICE)," Optics Express, vol. 18, no. 21.

[42] F. Paolucci, F. Cugini, A. Giorgetti, N. Sambo, and P. Castoldi, "A survey on the path computation element (PCE) architecture," Communications Surveys Tutorials, IEEE, vol. 15, no. 4, 2013.

[43] M. Secondini, E. Forestieri, and G. Prati, "Achievable information rate in nonlinear WDM fiber-optic systems with arbitrary modulation formats and dispersion maps," J. Lightwave Technol., 2013. 